\title{
Unified nonlinear theory of spontaneous and forced helical resonant MHD states
}

\author{
J. Loizu and P. Helander \\ Max-Planck-Institut für Plasmaphysik, D-17491 Greifswald, Germany
}

(Dated: March 3, 2017)

\begin{abstract}
It is shown that the theory of the nonlinearly saturated ideal internal kink mode by Rosenbluth, Dagazian, and Rutherford [Phys Fluids 16(11), 1894 (1973)] can be used to find the fully nonlinear ideal plasma response to an externally applied resonant magnetic perturbation. It is also demonstrated that the solution leads to a jump in the rotational transform across the resonant surface caused by a zonal current sheet. Its amplitude scales linearly with the plasma perturbation despite the nonlinearity of the solution. This confirms a recent conjecture that three-dimensional MHD equilibria with nested magnetic surfaces generally contain discontinuities in the rotational transform [J. Loizu, S. R. Hudson, et al, Phys Plasmas 22(9), 090704 (2015)]. It also lends support to Parker's long-standing suggestion that "almost all" MHD equilibria possess current sheets.
\end{abstract}

PACS numbers: 52.35.Ra, 52.35.Kt, 52.65.Ff

The theory of ideal magnetohydrodynamics (MHD) has no inherent length scale and thus allows current sheets to develop and cause discontinuities in the magnetic field [1-3]. Mathematically, these current sheets are represented by Dirac $\delta$-function current densities and are physically acceptable in the sense that their integral is finite. Sometimes it is argued that any finite resistivity, however small, will regularize the current density singularity, whose existence is therefore merely an academic matter. However, the mere tendency to form a true singularity in the ideal-MHD limit has fundamental and broader consequences for reconnection and turbulent dynamics across plasma physics [4-7].

For half a century, a quandary has existed at the heart of theoretical plasma physics: three-dimensional MHD equilibria with nested surfaces seem to suffer from two intolerable pathologies: (i) pressure-driven infinite currents arise around resonant rational surfaces unless the pressure gradient vanishes locally [8, 9] (at every resonant surface!); and (ii) the equilibrium field is not an analytical function of the shape of the boundary, since arbitrarily small perturbations can (even linearly) result in an unphysical overlapping of surfaces [10, 11]. Historically, the cause of these pathologies has been attributed to the class of possible pressure profiles, but the form of the pressure profile - whether it be smooth, continuous, or pathological in some sense - is not the cause of the problem, because the problem (ii) remains at zero pressure.

Recently, a new class of three-dimensional ideal-MHD equilibria with nested surfaces was proposed which allows for arbitrary pressure profiles, including smooth ones $[11,12]$. For this class of equilibria, all current densities are integrable and nested surfaces are preserved. These properties are ensured by the presence of zonal (i.e. netcurrent carrying) current sheets that produce a jump in the rotational transform across resonant surfaces. Since this seems to be the only class of equilibria that solves both problems (i) and (ii), it was conjectured that any physically valid equilibrium with nested resonant surfaces must belong to this class.
In this Letter, we show that the nonlinearly saturated internal kink mode [1] belongs to this new class of equilibria, i.e., that the rotational transform jumps across the resonant surface and does so with a magnitude that scales as predicted by the conjecture. We also demonstrate that the same theory can be applied to find the fully nonlinear, ideal plasma response to an externally applied resonant magnetic perturbation (RMP), which thus causes a discontinuity in the rotational transform at the rational surface. This result unifies the description of spontaneous and forced helical equilibrium states and confirms the conjecture for a large-aspect-ratio tokamak. It also elucidates the question of how to reliably predict the ideal plasma response to RMPs [13], which is of crucial importance for present and future magnetic fusion devices [14].

The need for a jump in the rotational transform, $\Delta t$, to develop in three-dimensional MHD equilibria with nested resonant surfaces follows from a sine qua non condition for the existence of perturbed equilibria that can be written as $|d \xi / d s|<1$, which states that the differential displacement of magnetic surfaces, $d \xi$, with respect to an initial equilibrium, cannot exceed the differential distance between surfaces, $d s=\sqrt{g_{\psi \psi}} d \psi$. Here $\psi$ is a general flux-surface label and $g_{\psi \psi}$ is the corresponding metric element. This condition guarantees that nested surfaces are preserved. From an asymptotic study of Newcomb's equation [15], which determines the radial profile of the displacement in a perturbed screw-pinch, a condition was derived [11] for the minimum magnitude of the jump in rotational transform across a resonant rational,

$$
\Delta t>\Delta t_{\min }=2 t_{s}^{\prime} \xi_{s}
$$

which guarantees $|d \xi / d r|<1$. Here $\xi_{s}$ and $t_{s}^{\prime}$ are the displacement and radial derivative of the rotational transform, respectively, both evaluated at the resonant surface. Note that this jump is associated with a zonal current sheet, by which we mean that the surface-average of the $\delta$-function current density is non-zero.

The $m=n=1$ internal kink instability occurs in a cylindrical tokamak when the safety factor is anywhere 
$q=1 / t<1$. As noted by Rosenbluth, Dagazian and Rutherford [1] in their theory of the nonlinear saturation of this instability, however, all harmonics are excited to comparable amplitude in a boundary layer around the $q=1$ surface, and therefore perturbation theory is not applicable. By using a full nonlinear treatment, a nearby ideally-stable equilibrium was found that has nested surfaces, an axisymmetric boundary, a current sheet on the resonant surface, and an inner helical plasma column with helicity $m=n=1$. The nonlinear solution for the displacement of the magnetic surfaces is

$$
\xi(x, \theta)=\int_{0}^{x}\left[\frac{\left|x^{\prime}\right|}{\sqrt{f\left(x^{\prime}\right)+g(\theta)}}-1\right] d x^{\prime}+h(\theta)
$$

where $\theta$ is the polar angle and $\xi$ is the radial displacement of a flux surface originally situated at a radius $x$ with respect to the resonant surface. The displacement is ensured to be continuous and is fully determined by three functions, namely $f(x), g(\theta)$, and $h(\theta)$. Before calculating these functions we notice that, thanks to Eq. (2), the equilibrium is guaranteed to satisfy the sine qua non condition, in fact, marginally:

$$
\lim _{x \rightarrow 0} \frac{\partial \xi}{\partial x}=-1
$$

The function $h(\theta)=\frac{1}{2} \xi_{a} \cos \theta$ is determined by asymptotic matching to linear theory outside the resonant layer, i.e. by imposing $\xi(x \rightarrow-\infty, \theta)=\xi_{a} \cos \theta$ and $\xi(x \rightarrow+\infty, \theta)=0$ in Eq. (2). Here $\xi_{a}$ is the kink of the magnetic axis, which is unknown a priori. The function $f(x)$ can be determined once $g(\theta)$ is known, since it satisfies

$$
\oint \frac{d \theta}{\sqrt{f(x)+g(\theta)}}=\frac{1}{x}
$$

where $\oint d \theta=\int_{0}^{2 \pi} d \theta / 2 \pi$. Equation (4) is simply the constraint (to lowest order in $\xi$ ) of conserved toroidal magnetic flux. The most complicated task is to determine $g(\theta)$, which is the solution to the integral equation

$$
\int_{0}^{\infty} d f \frac{2 u^{\prime}(f)}{u^{3}(f)}\left[\frac{1}{\sqrt{f+\hat{g}(\theta)}}-u(f)\right]=\cos \theta
$$

where $\hat{g}=g / \xi_{a}^{2}$ and $u(f)=\oint d \theta / \sqrt{f+\hat{g}(\theta)}$. Fortunately, Rosenbluth could reformulate this equation as a variational principle, in which $g(\theta)$ is the function that extremizes the functional

$$
\Lambda[\hat{g}]=\int_{0}^{\infty} d f\left[\frac{1}{u(f)}-\sqrt{f}-\frac{1}{2 \sqrt{f}} \oint \hat{g} d \theta\right]+\oint \hat{g} \cos \theta d \theta
$$

As a matter of fact, $\Lambda$ possesses a maximum, $\Lambda_{\max } \approx$ 0.025, which determines the amplitude of the kink, $\xi_{a}$ [1]. In order to maximize $\Lambda$, we rewrite the first integral in Eq. (6) in a way that avoids subtracting two divergent terms, namely the first two terms as $f \rightarrow \infty$. Then, the extremum is found numerically by writing $g(\theta)=$ $\left(\sum c_{m} \cos (m \theta)\right)^{2}$ and iterating on $\left\{c_{m}\right\}$. The quadratic form is used to ensure that $g \geq 0$. Figure 1 shows the solution for the functions $g(\theta)$ and $f(x)$ as well as the corresponding displacement $\xi(x, \theta)$.

First, we observe the formation of a nonlinear layer in $\xi$ around the resonant surface. The width of the layer, $l$, depends on $\theta$, with a maximum scale $l \sim O\left(\xi_{a}\right)$ at $\theta=0$, and no minimum scale, $l \rightarrow 0$ as $\theta \rightarrow \pi$. This type of solution is expected by virtue of Eq. (3). In fact, for $\theta>\pi / 2$ the displacement must go from $\xi(x \rightarrow-\infty, \theta)=$ $\xi_{a} \cos \theta<0$ to $\xi(x \rightarrow+\infty, \theta)=0$ while developing a negative gradient, Eq. (3), at the resonant surface. Hence a nonlinear layer, at least cubic in $x$, must form around the resonant surface.

Second, we remark that the function $g(\theta)$ can be very well approximated by

$$
g(\theta) \approx \frac{1}{3} \xi_{a}^{2} \cos ^{8}(\theta / 2) .
$$

This is a universal solution in the sense that it does not depend on the specific equilibrium current and pressure profiles. Its form is inferred from an asymptotic analysis, and the coefficient in front has been adjusted to match the maximum of the function to the numerical solution. We now verify that this form for $g$ is consistent with the asymptotics of Eq. (5) for $\theta \rightarrow \pi$. Taking the derivative of Eq. (5) with respect to $\theta$, we have that

$$
\int_{0}^{\infty} \frac{u^{\prime}(f)}{u^{3}(f)} \frac{d f}{[f+\hat{g}(\theta)]^{3 / 2}} \hat{g}^{\prime}(\theta)=\sin \theta
$$

and for small $g$, the integral is dominated by contributions from small $f$. Assuming that around $\theta=\pi$ we have $\hat{g}(\theta) \sim \lambda(\pi-\theta)^{n}$, we find that $u(f) \sim c_{n} \lambda^{-1 / n} f^{1 / n-1 / 2}$ for $f \rightarrow 0$, where $c_{n}=\int_{0}^{\infty}\left(1+x^{n}\right)^{-1 / 2} d x$. Thus Eq. (8) is, for $\theta \rightarrow \pi$,

$$
\left(\frac{n}{2}-1\right) \frac{\sqrt{\lambda}}{c_{n}^{2}}(\pi-\theta)^{\frac{n}{2}-3} d_{n}=\pi-\theta
$$

where $d_{n}=\int_{0}^{\infty} x^{2 / n}\left(1+x^{n}\right)^{-3 / 2} d x$. Hence $n=8$ and $\lambda=c_{n}^{4} /\left(9 d_{n}^{2}\right)$, which can be evaluated using Gamma functions, giving $\lambda \simeq 10^{-3}$. The expansion of Eq. (7) around $\theta=\pi$ gives $\hat{g}(\theta) \sim(\pi-\theta)^{8} / 768$, in reasonable agreement with the asymptotic calculation.

Finally, we remark that while the displacement, $\xi$, is continuous, the poloidal magnetic field, $B_{\theta}$, is not. A jump occurs across the resonant surface [6],

$$
\left[\left[B_{\theta}\right]\right]=2 \frac{r_{s}}{R} B_{z} t_{s}^{\prime} \xi_{a} \sqrt{g(\theta) / \xi_{a}^{2}}
$$

where $\left[\left[B_{\theta}\right]\right]=B_{\theta}\left(x=0^{+}\right)-B_{\theta}\left(x=0^{-}\right), r_{s}$ is the initial radius of the resonant surface, $2 \pi R$ is the length of the periodic cylinder, $B_{z}$ is the axial or toroidal magnetic field, and $t_{s}^{\prime}$ is the radial derivative of the equilibrium rotational transform, $t=R B_{\theta} /\left(r B_{z}\right)$, at $r=r_{s}$. Equations (7) and (10) imply the existence of a current sheet 
peaking at $\theta=0$ and with multiple harmonic content: $m=0, m=1$, and $m=2$ components are present. The finite zonal component $(m=0)$ is what produces a jump in the rotational transform,

$$
\Delta t=2 t_{s}^{\prime} \xi_{a} \oint \sqrt{g(\theta) / \xi_{a}^{2}} d \theta \approx 0.45 t_{s}^{\prime} \xi_{a}
$$

which, despite the nonlinearity of the solution, scales linearly with the axis kink! The scaling in Eq. (11) agrees with the constraint given by Eq. (1), but the coefficient in front is slightly different because in Eq. (1), $\Delta t$ refers to the jump across the entire nonlinear layer whereas in Eq. (11) it only denotes the actual discontinuity at $x=0$. There is, in fact, a nonsingular part of the current density within the boundary layer that accounts for the other half of the total zonal current [3]. In any case, this calculation proves that the saturated internal kink contains a discontinuity in the rotational transform and thus belongs to the class of equilibria postulated in Ref. [11].

The nonlinear theory developed by Rosenbluth et al. is in fact more general than previously recognized. All that the theory does is to seek helical states of given helicity $(m, n)$ that satisfy ideal force-balance inside and outside a boundary layer around a resonant rational surface. This is carried out by applying flux-conservation constraints with respect to an initially axisymmetric state, solving the force-balance equation in the nonlinear layer and matching the solution to linear theory outside the layer. With this in mind, one can reformulate the problem for a forced helical state produced by applying a resonant magnetic perturbation on the boundary of a cylindrical tokamak. For a generic boundary perturbation of the form $\xi_{a} \cos (m \theta-n \varphi)$, it follows that Eqs. (2), (3) and (4) are still valid, and Eqs. (5) and (6) are only slightly modified, simply because the helicity is general and the perturbation is nonzero on the boundary and zero on the axis rather than the other way around. In particular, the functional $\Lambda[\hat{g}]$ becomes

$\Lambda[\hat{g}]=\int_{0}^{\infty} d f\left[\frac{1}{u(f)}-\sqrt{f}-\frac{1}{2 \sqrt{f}} \oint \hat{g} d \theta\right]-\oint \hat{g} \cos (m \theta) d \theta$.

The maximum of $\Lambda$ is found to be independent of $m$ and has the same value as for the internal kink. The numerical solution for the function $g$ is again well approximated by

$$
g(\theta) \approx \frac{1}{3} \xi_{a}^{2} \sin ^{8}(m \theta / 2)
$$

and the solutions for $f(x)$ and $\xi(x, \theta)$ are the same as before with the replacements $x \rightarrow-x$ and $\theta \rightarrow m \theta+\pi / 2$. We would like to remark that the implicit $\varphi$-dependence in Eq. (13) is obtained by replacing $m \theta$ with $m \theta-n \varphi$, as for the internal kink.

An example of nonlinear solution for an $m=2, n=1$ boundary perturbation is shown in Fig. 2. For this case, the nonlinear layer becomes infinitesimally narrow at two poloidal locations, but only one is shown. A jump in rotational transform is also present in the nonlinear solution and is given by

$$
\Delta t=2 m t_{s}^{\prime} \xi_{a} \oint \sqrt{g(\theta) / \xi_{a}^{2}} d \theta \approx 0.9 t_{s}^{\prime} \xi_{a} .
$$

The jump in rotational transform obtained for a general RMP is thus the same as for the saturated internal kink, except for an additional linear scaling with the poloidal mode number $m$ and the fact that the quantity $\xi_{a}$ is the amplitude of the RMP, which is thus known.

There are several important implications of these results for both theory and experiments. First, we have confirmed the conjecture that MHD equilibria with nested and resonant surfaces possess discontinuous rotational transform, i.e. zonal current sheets, across the resonances, by showing that this statement is rigorously true in a large-aspect-ratio tokamak. An important consequence is that this class of equilibria can support arbitrarily smooth pressure profiles without generating infinite currents. The discovery of these equilibria lends support to Parker's long-standing suggestion that "almost all" MHD equilibria possess current sheets [16, 17]. Second, an explanation emerges for the apparent disagreement between linear and nonlinear ideal equilibrium codes when trying to calculate the plasma response to RMPs in tokamaks [10]: on the one hand, linear codes allow for current sheets to form, but as of now they have always assumed that the zonal component of the current sheet vanishes (thus leading to an unphysical overlapping of surfaces and the need to artificially flatten the pressure to avoid infinite pressure-driven currents); on the other hand, nonlinear codes are by construction guaranteed to preserve the topology of nested surfaces, but do not allow magnetic field discontinuities (thus the current sheet is never resolved). Third, the presence of a zonal current sheet has a global effect on the plasma response: RMPs were recently predicted to penetrate beyond the resonance and to be significantly amplified with increasing plasma pressure [12]. This prediction was based on the conjecture that has now been proved, but did not provide a specific value for the jump in rotational transform, which is now supplied by the nonlinear theory. Remarkably, perhaps, the linear scaling of the rotational transform with the RMP amplitude remains valid in the nonlinear theory. The latter also makes a further prediction, namely, that higher harmonics are excited to comparable amplitude throughout the entire plasma column enclosed by the resonant surface (even without any toroidicity-induced mode coupling). Finally, we note that, by generalizing the nonlinear theory discussed here to non-circular and toroidal geometry, quantitative predictions can be produced for the ideal plasma response to RMPs in tokamaks.

We acknowledge very helpful discussions with Ami- 
tava Bhattacharjee, Stuart Hudson and Harold Weitzner. This work has been carried out in the framework of the EUROfusion Consortium and has received funding from the Euratom research and training programme 2014-2018 under grant agreement No 633053. The views and opinions expressed herein do not necessarily reflect those of the European Commission.
[1] M. N. Rosenbluth, R.Y. Dagazian, and P. H. Rutherford. Nonlinear properties of the internal $\mathrm{m}=1$ kink instability in the cylindrincal tokamak. Phys. Fluids, 16(1973):1-30, 1973.

[2] T. S. Hahm and R. M. Kulsrud. Forced magnetic reconnection. Physics of Fluids, 28(8):2412, 1985.

[3] Allen H. Boozer and Neil Pomphrey. Current density and plasma displacement near perturbed rational surfaces. Physics of Plasmas, 17(11), 2010.

[4] C. S. Ng and A. Bhattacharjee. Nonequilibrium and current sheet formation in line-tied magnetic fields. Physics of Plasmas, 5(11):4028-4040, nov 1998.

[5] F. L. Waelbroeck. Current sheets and nonlinear growth of the $\mathrm{m}=1$ kinktearing mode. Physics of Fluids B: Plasma Physics (1989-1993), 1(12):2372-2380, 1989.

[6] L. Zakharov, B. Rogers, and S. Migliuolo. The theory of the early nonlinear stage of $\mathrm{m}=1$ reconnection in tokamaks. Physics of Fluids B: Plasma Physics, 5(7):24982505, 1993.

[7] A. Bhattacharjee, T. Hayashi, C. C. Hegna, N. Nakajima, and T. Sato. Theory of pressure-induced islands and self-healing in three-dimensional toroidal magnetohydrodynamic equilibria. Physics of Plasmas, 2(3):883, 1995.

[8] H. Grad. Toroidal Containment of a Plasma. Physics of Fluids, 10(1):137, 1967.

[9] P. Helander. Theory of plasma confinement in nonaxisymmetric magnetic fields. Reports on Progress in Physics, 77(8):087001, aug 2014.

[10] A. D. Turnbull, N. M. Ferraro, V. A. Izzo, E. A. Lazarus, J.-K. Park, W. A. Cooper, S. P. Hirshman, L. L. Lao, M. J. Lanctot, S. Lazerson, Y. Q. Liu, A. Reiman, and F. Turco. Comparisons of linear and nonlinear plasma response models for non-axisymmetric perturbations. Physics of Plasmas, 20(5):056114, may 2013.

[11] J. Loizu, S. Hudson, A. Bhattacharjee, and P. Helander. Magnetic islands and singular currents at rational surfaces in three-dimensional magnetohydrodynamic equilibria. Physics of Plasmas, 22(2):022501, feb 2015.

[12] J. Loizu, S. R. Hudson, P. Helander, S. A. Lazerson, and A. Bhattacharjee. Pressure-driven amplification and penetration of resonant magnetic perturbations. Physics of Plasmas, 23(5), 2016.

[13] Yueqiang Liu, C J Ham, A Kirk, Li Li, A Loarte, D A Ryan, Youwen Sun, W. Suttrop, Xu Yang, and Lina Zhou. ELM control with RMP: plasma response models and the role of edge peeling response. Plasma Physics and Controlled Fusion, 58(11):114005, 2016.

[14] T. E. Evans, R. a. Moyer, P. R. Thomas, J. G. Watkins, T. H. Osborne, J. a. Boedo, E. J. Doyle, M. E. Fenstermacher, K. H. Finken, R. J. Groebner, M. Groth, J. H. Harris, R. J. La Haye, C. J. Lasnier, S. Masuzaki, N. Ohyabu, D. G. Pretty, T. L. Rhodes, H. Reimerdes, D. L. Rudakov, M. J. Schaffer, G. Wang, and L. Zeng. Suppression of Large Edge-Localized Modes in HighConfinement DIII-D Plasmas with a Stochastic Magnetic Boundary. Physical Review Letters, 92(23):235003, jun 2004.

[15] William A. Newcomb. Hydromagnetic stability of a diffuse linear pinch. Annals of Physics, 10(2):232-267, 1960.

[16] E. N. Parker. Topological dissipation and the smallscale fields in turbulent gases. The Astrophysical Journal, 174:499-510, 1972.

[17] E. N. Parker. Spontaneous current sheets in magnetic fields. Oxford University Press, 1994. 

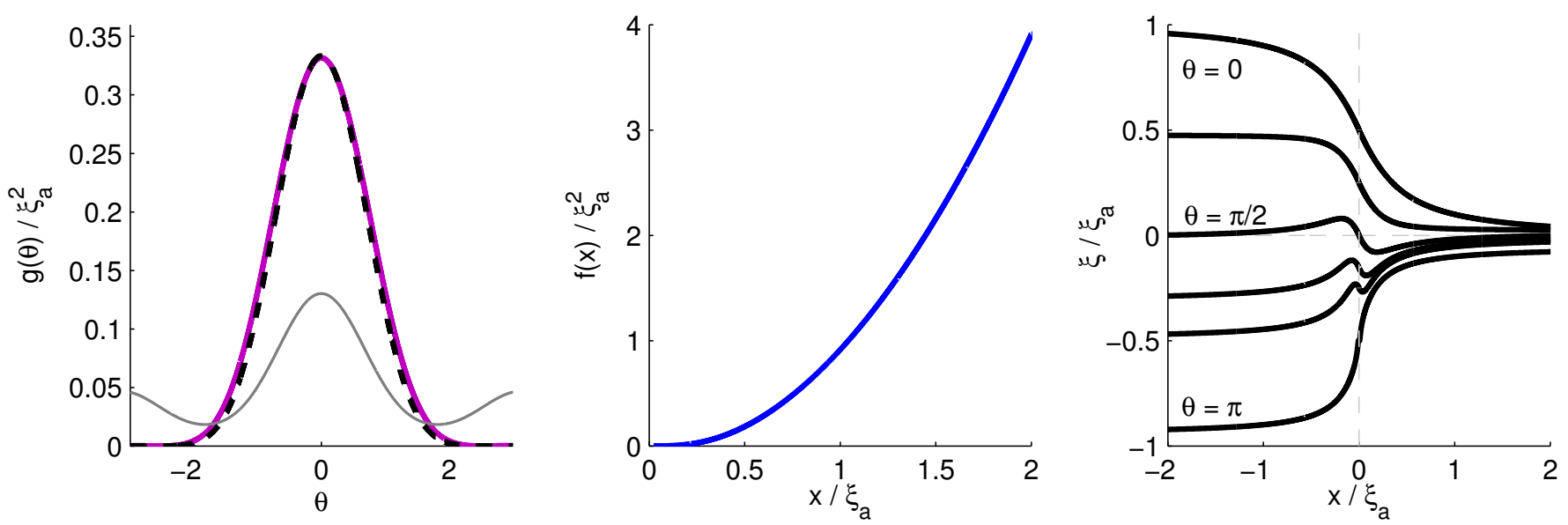

FIG. 1: Nonlinear solution for the internal kink. Left: numerical solution for $g(\theta)$ (solid magenta) obtained by minimizing the functional $\Lambda$, Eq. (6), starting from an initial guess (solid grey). The dashed-black line is the function $\frac{1}{3} \cos ^{8}(\theta / 2)$. Middle: numerical solution for $f(x)$ obtained from Eq. (4) and using the solution for $g(\theta)$. Right: numerical solution for the radial profile of the displacement at different poloidal locations. The location of the resonant surface is $x=0$.
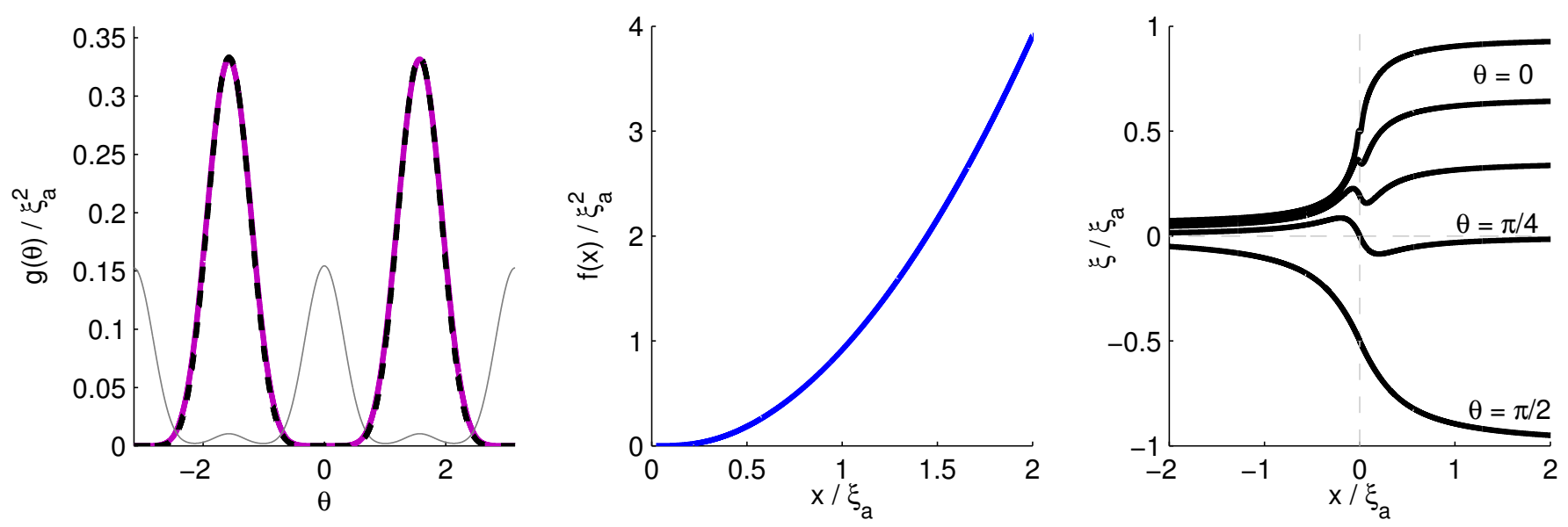

FIG. 2: Nonlinear solution for an externally forced helical state with helicity $m=2, n=1$. Left: numerical solution for $g(\theta)$ (solid magenta) obtained by minimizing the functional $\Lambda$, Eq. (12), starting from an initial guess (solid grey). The dashed-black line is the function $\frac{1}{3} \sin ^{8}(\theta)$. Middle: numerical solution for $f(x)$ obtained from Eq. (4) and using the solution for $g(\theta)$. Right: numerical solution for the radial profile of the displacement at different poloidal locations. The resonant surface is at $x=0$. 